\title{
Revisión de la utilidad de diferentes innovaciones docentes: elementos dinamizadores, evaluación formativa y desarrollo de competencias transversales.
}

\section{Fernando Cardona $^{a, b}$, Juan Antonio Llorens-Molina ${ }^{c}$}

${ }^{a}$ Departamento de Tecnología de Alimentos. Escuela Técnica Superior de Ingeniería Agronómica y del Medio Natural. Universitat Politècnica de València. Spain. ${ }^{\mathrm{b}}$ Instituto de Biomedicina de Valencia. Consejo Superior de Investigaciones Científicas. fcardona@ibv.csic.es ${ }^{\mathrm{c}}$ Instituto Agroforestal Mediterráneo. Escuela Técnica Superior de Ingeniería Agronómica y del Medio Natural. Universitat Politècnica de València. Spain.

\section{Resumen}

Durante varios cursos académicos se han ido implantando diversas innovaciones docentes en la asignatura de Fundamentos de Química para Ciencia y Tecnología de los Alimentos. Entre otros, se ha utilizado el aprendizaje basado en proyectos, mediante presentación de trabajos en persona u on-line mediante screencast, la gamificación para evaluación y repaso de conceptos, y los exámenes on-line como método de evaluación formativa y de feedback rápido. Por otro lado, la implementación gradual de la docencia inversa ha sido otra de las mejoras introducidas, dado que es la tendencia actual en educación, y en concreto, en la educación superior.

Tras varios años de implementación de estas metodologías, cabe echar la vista atrás y revisar los resultados que han proporcionado, con el objetivo de implantarlas definitivamente, descartarlas totalmente o corregir los defectos de implementación que hayan podido surgir, y cuya corrección pueda servir para correcta implantación.

En algunos casos, estas innovaciones han presentado ventajas claras para los alumnos y profesores. En otros casos, pese a presentar ventajas, han aumentando considerablemente la carga de trabajo del profesorado o del alumnado. En la mayoría de casos estas actividades gustan a los alumnos, ya que ayudan a dinamizar las clases y al enfoque práctico de la asignatura. Al profesorado, como norma, le permite evaluar competencias que no son evaluables con el resto de actividades académicas, como las competencias trasnversales. Además, este tipo de actividades también sirven para afianzar y aplicar los conceptos teóricos estudiados de una manera más activa. 
Palabras clave: Innovación educativa, Aprendizaje Basado en Proyectos, Screencast vídeos, Competencias Transversales, Casos de estudio, Gamificación, Cuestionarios on-line, Evaluación formativa, Docencia Inversa

\section{Introducción}

Las tendencias actuales en educación incluyen una serie de metodologías que no siempre son fáciles de implementar, requiriendo experiencias piloto previas a su implantación, de forma que pueda evaluarse si presentan beneficios en el proceso de enseñanza-aprendizaje (E-A). De esta forma se puede medir si realmente presentan beneficios académicos, así como los costes de su implantación a nivel de esfuerzo en profesores y alumnos. Además, utilizando estas experiencias piloto pueden corregirse errores de implantación debido al desconocimiento de la metodología o un exceso en las expectativas de resultados de las mismas. Estos ensayos previos premiten también conocer su utilidad para el desarrollo de competencias transversales, que es un proyecto prioritario en muchas universidades europeas (Universitat Politécnica de Valencia, http://www.upv.es/contenidos/COMPTRAN/). En el caso de la docencia inversa (DI), resulta casi imprescindible implantarla en varios cursos académicos, dada la elevada carga de trabajo que conlleva, y lo arriesgado, en lo que a resultados se refiere.

En lo relativo al aprendizaje basado en proyectos (ABP), se ha demostrado útil como metodología de E-A (Rodríguez-Sandoval y col., 2010), además de que la identificación de los conceptos químicos teóricos con situaciones reales en el campo de trabajo (aprendizaje en contexto, AEC), ayuda a la comprensión de los mismos, y mejora la motivación de los alumnos (De Jong, 2006). Mediante esta metodología, pueden desarrollarse y evaluarse varias competencias transversales (Cardona y Atarés, 2016), aunque algunas de ellas, al igual que el aprendizaje cooperativo, solamente si se realizan en grupos (Durán-Aponte y Durán-García, 2013). Las Competencias Transversales (CTs) son aquellas necesarias en un graduado, pero no necesariamente relacionadas con los conocimientos específicos de su disciplina (http://www.upv.es/contenidos/COMPTRAN/). Por lo tanto, una CT podría definirse como "la capacidad de lograr un objetivo o resultado en un entorno de aplicación real, que no está directamente relacionada con conocimientos técnicos". Así pues, parece lógico utilizar aplicaciones reales (enseñanza "en contexto") para desarrollarlas y para evaluarlas.

Por otro lado, la evaluación formativa puede basarse en diferentes recursos, tanto presenciales como on-line. La mejora del rendimiento académico que se obtiene al realizar la evaluación formativa mediante esta metodología es un hecho constatado en asignaturas 
básicas al inicio de la educación superior (Parker y Loudon, 2012). Según Sadler y Good, (2006), la autoevaluación contribuye a la mejora del aprendizaje a través de diferentes aportaciones: pedagógicas, por retroalimentación; metacognitivas, por la mayor consciencia del estudiante de sus limitaciones, potencialidades y evolución; de carácter práctico, por suponer un importante ahorro de tiempo para el profesorado; y afectivas, ya que el alumno la percibe como instrumento de aprendizaje y no sancionador. Sin embargo, hay múltiples aspectos del diseño de la evaluación formativa que son susceptibles de análisis y mejora, siendo un dato especialmente valioso la opinión de los estudiantes sobre la actividad.

La DI es la tendencia actual en educación, y en concreto en la educación superior (Uskoković, 2018). Tradicionalmente, la docencia se ha basado en la clase magistral seguida de su revisión y aplicación de forma no presencial. Por el contrario, en la DI, parte de la información se transmite de forma no presencial mediante objetos de aprendizaje que el alumno trabaja de manera autónoma, previamente a las tareas presenciales, de mayor complejidad y demanda cognitiva. De este modo, el alumno adquiere mayor tasa de responsabilidad en el proceso E-A. Los resultados de la DI han sido evaluados en numerosoas investigaciones, algunas de ellas relacionadas con el aprendizaje de la química (Ryan y Reid, 2015), así como con las prácticas de laboratorio (Teo y col., 2014). En general, se confirman los efectos positivos en el rendimiento académico y en la motivación e implicación de los estudiantes. En cuanto a las prácticas de laboratorio, Teo y col. (2014), mediante vídeos y cuestiones prelaboratorio, consiguen la comprensión de la teoría y los objetivos experimentales, además del ahorro de tiempo al prescindir de la introducción teórica en el laboratorio.

Pese al balance generalmente positivo de la DI, también existen dificultades descritas en la literatura (Akçayir y Akçayir, 2018); la necesidad de adaptación de los materiales didácticos supone un esfuerzo importante al profesorado, sobre todo los primeros años. Desde el punto de vista de los estudiantes, existe diferente grado de implicación en la preparación del trabajo no presencial (Jensen y col., 2018). Además, es especialmente difícil la adaptación a la DI del alumnado de primer curso de estudios universitarios (O’Flaherty y Phillips, 2015), debido a la baja capacidad de trabajo autónomo en etapas inciales de la educación superior.

Tras varios años de implementación de estas metodologías, cabe echar la vista atrás y revisar los resultados que han proporcionado a alumnos y docentes, con el objetivo de implantarlas definitivamente, descartarlas totalmente o corregir los defectos de implementación que hayan podido surgir con el objetivo de implantarla correctamente. 


\section{Objetivo}

El objetivo general de este trabajo es conocer la utilidad de las mejoras docentes implementadas, comparándolas entre sí. Los resultados permitirán conocer en cuáles se debe focalizar los esfuerzos, y cuáles no tienen potencial de mejora en este contexto.

\section{Contexto}

Las innovaciones o mejoras docentes objeto de esta revisión se han realizado en la asignatura Fundamentos Químicos para la Ciencia y Tecnología de Alimentos de 12 créditos ( 9 teóricos y 3 prácticos), de primer curso del grado en Ciencia y Tecnología de los Alimentos, de la Universitat Politecnica de València.

\section{Desarrollo}

Para el aprendizaje basado en proyectos, los trabajos se han presentado en persona u on-line mediante screencast (software Screencast-O-Matic, https://screencast-o-matic.com/), la gamificación se ha realizado mediante Socrative (https://socrative.com/) y los exámenes online en Poliformat (https://poliformat.upv.es/). La docencia inversa se ha implementado gradualmente. La opinión de los alumnos se ha sondeado utilizando formularios de Google (https://www.google.es/intl/es/forms/about/).

A continuación, se revisarán los resultados obtenidos con cada una de ellas, identificando los puntos de mejora.

\section{a. Introducción de metodologías constructivistas.}

La aproximación incluye la introducción de ABP-AEC mediante ejemplos reales de la industria alimentaria durante las clases magistrales, además de la realización de un trabajo por parte de los alumnos sobre este tipo de aplicaciones, que puede subir la calificación pero no es obligatorio realizarlo, ni necesario para obtener la calificación máxima. Durante varios años de ha implantado esta metodología, obteniéndose en todos los cursos resultados similares, pero permitiendo corregir algunos problemas de su implantación.

En Cardona y Atarés (2016), se concluyó que la realización del trabajo conseguía aumentar la motivación de los alumnos y las calificaciones de la parte práctica, además de resultar de utilidad para el desarrollo y evaluación de varias CTs. En Cardona y Atarés (2017), los 
resultados mostraron mejoría a nivel de motivación y calificaciones, si bien se observa que generan una sobrecarga de trabajo en el alumnado de primer curso, sobre todo debido al elevado nivel de los trabajos y a la falta de conocimientos previos sobre el tema. En Cardona y Rubio-Granero (2018), de nuevo se observa mejora en las calificaciones de la parte práctica y la utilidad para evaluar CTs. Sin embargo, a pesar de que lo realizan sin ser obligatorio, se percibe desagrado por parte de los estudiantes, principalmente por el hecho de tener que presentar el trabajo en el aula delante de los compañeros y el profesor, además del elevado nivel de los trabajos (a pesar de haberse rebajado respecto al año anterior). En este curso se ofrece la opción de realizar la presentación mediante screencast, pero manteniendo el turno de preguntas de los compañeros después de reproducir las presentaciones en clase. Cabe decir que la mayoria de los alumnos eligió hacer la presentación por screencast, y aproximadamente la mitad lo hizo por evitar hablar en público, lo que apunta la necesidad de incidir en esa competencia (Comunicación oralefectiva), de elevada importancia en la preparación de los estudiantes. Por estos motivos, se concluye que, aunque la presentación por screencast permite igualmente evaluar esta competencia (presentación acompañada de imagen), podría ser menos útil a la hora de desarrollarla. Por su parte, Llorens-Molina y Llorens de Jaime (2018), coinciden de nuevo en el efecto motivador, en la mejora del proceso de aprendizaje y en el desarrollo de CTs al realizar las presentaciones mediante screencast. Además, al introducir la evaluación por pares no encuentran diferencias entre las calificaciones de los profesores y las de los alumnos, siendo más formativo, ya que los alumnos tienen que estar más atentos a las presentaciones de los compañeros para evaluarlas. En cuanto a la rúbrica proporcionada a los alumnos para la evaluación por pares, se utilizó una generada previamente para evaluar presentaciones de trabajos de fin de grado (Verdeguer y col., 2017). Aunque en líneas generales parece útil para los alumnos, existen algunos elementos de mejora, siendo algunos ítems poco operativos para los alumnos. En Llorens-Molina y Cardona (2019), esta actividad de nuevo resulta útil para desarrollar y evaluar CTs, pero apunta problemas en las habilidades de los alumnos para la búsqueda de información para realizar la tarea, probablemente debido a que son de primer curso. Ademas, dado que en este curso se realizaron mediante presentación por screencast y evaluación por pares, se observaron problemas con la rúbrica proporcionada a los alumnos, además de que se muestra como necesaria la discusión de los trabajos, ya sea on-line o de modo presencial, para que se preste atención a las presentaciones. En cuanto a la rúbrica proporcionada a los alumnos para la evaluación por pares, aunque mejorada respecto al año anterior, siguen existiendo puntos de mejora en cuanto a operatividad y claridad de algunos items.

En todos los casos la introducción de ejemplos reales de la industria alimentaria durante las clases magistrales ha sido percibido por los alumnos como útil y agradable, y parece tener un efecto motivador, dado que se obtienen mejores calificaciones en la parte aplicada de la asignatura. Por estos motiovs, no se han encontrado puntos de mejora en esa parte. 


\section{b. Evaluación formativa.}

En lo referente a la evaluación formativa, se han realizado aproximaciones relacionadas con la autoevaluación, la ludificación, la evaluación on-line y la evaluación por pares.

Llorens-Molina (2017) concluye que la autoevaluación on-line tiene problemas importantes como evaluación sumativa, aunque sí es útil para el proceso de aprendizaje, sobre todo para que el alumno evalúe su evolución y nivel de conocimientos para preparar las pruebas de evaluación. Por su parte, Cardona y Rubio-Granero (2018) encuentran que la ludificación de los cuestionarios mediante Socrative, realizada de forma sumativa, es bien acogida por los estudiantes pero no mejora significativamente los resultados académicos, y genera una excesiva carga de trabajo al profesorado en la versión gratuita del software. En cuanto al uso de exámenes on-line, presentaron una excelente acogida por los estudiantes, principalmente por el feedback y la calificación en tiempo real, por lo que no se han encontrado elementos de mejora, más allá de la dificultad del control del plagio, de fácil solución.

\section{c. Docencia inversa.}

En cuanto a la implantación de la DI, es probablemente que suponga mayor esfuerzo del profesorado, y en general es peor valorada por los estudiantes. Llorens-Molina (2017) observa que las grabaciones de las lecciones mediante screencast y videos son bien valorados como objetos de aprendizaje, aunque la forma en que se utilizan no se ajusta siempre al objetivo planteado, y son empleados como material de consulta y revisión para la preparación de exámenes. Además, deberían dotarse de mayor interactividad y de una relación más explícita con las actividades ordinarias de clase, en vistas a la transición a la clase inversa (Llorens-Molina , 2017b). Cardona y Llorens-Molina (2019) concluyen que en general la DI es valorada positivamente en cuanto a los recursos utilizados: material audiovisual, plataforma educativa y autoevaluaciones, ya desde el primer año de implantación. Sin embargo, la percepción global de la DI por los alumnos no es buena, por lo que probablemente sea necesario explicar mejor al alumnado la metodología, así como proporcionarle pautas y orientaciones para mejorar la organización y gestión del tiempo (CT). Además, para obtener un impacto neto positivo seria necesaria, si no su aplicación en todas las asignaturas de un curso, al menos una coordinación entre ellas que evite interferencias en el trabajo no presencial.

En lo referente la implantación de la DI en las prácticas de laboratorio, Llorens-Molina (2008) implantó de manera experimental actividades previas en lugar de la introducción de la misma en el laboratorio. Estas actividades consistían en la visualización de un vídeo explicativo, seguido de un test para determinar si se habían asimilado los conceptos. Los resultados fueron comparables al método tradicional, en lo referido a calificaciones y motivación. El único problema observado en algunas ocasiones en las actividades pre- 
laboratotio fue la falta de comprensión global del objetivo de la práctica, y de su sentido dentro de la asignatura (Llorens-Molina y col., 2014). Además, estas actividades son capaces de potenciar interés por la investigación en los alumnos si se usan de forma correcta, utilizando actividades de nivel cognitivo alto, y no solo cuestiones técnicas o de manipulación. Por otro lado, Llorens-Molina y Cardona (2019) observaron que mejora el rendimiento académico, pero los alumnos prefieren la metodología tradicional, probablemente porque requiere menor esfuerzo autónomo. Sí que fueron bien acogidas las actividades pre-laboratorio, y además los estudiantes prefieren realizar las cuestiones previas, el informe de laboratorio y el examen, on-line, frente a hacerlo en papel.

\section{Conclusiones}

a. En cuanto a la introducción de metodologías contructivistas, puede concluirse que tiene efectos positivos en la motivación, en el aprendizaje, y en desarrollo de CTs. Algunos aspectos de mejora observados son relativos al nivel de exigencia sobre los alumnos: nivel de los contenidos, carencia de algunos conocimientos previos y carga de trabajo generada. La presentación de los trabajos mediante screencast permite la evaluación de algunas CTs, pero es menos efectiva en el desarrollo de la CT “comunicación oral". Algunas CTs solo pueden desarrollarse y evaluarse si los trabajos se realizan en grupo.

b. En lo relativo a la evaluación formativa, la autoevaluación y la ludificación solo son adecuadas como elemento formativo, no sumativo. Los exámenes on-line sí parecen adecuados también para la evaluación sumativa. Mediante evaluación por pares se obtienen resultados similares a los de la tradicional, y es bien aceptada por los estudiantes.

c. La implementación de la docencia inversa obtiene en general buenos resultados académicos, pero a costa de aumentar la carga de trabajo para profesorado y alumnado. Esto hace que tenga mala aceptación entre los alumnos, reticentes a trabajar de manera más autónoma y asumir más responsabilidad en su educación, al menos en el primer curso. Aunque su implantación parece viable en prácticas de laboratorio, en la teoría pueden generarse problemas, a no ser que se implante de forma generalizada, evitando así las comparaciones con los otros métodos de docencia.

\section{Referencias}

Akçayır, G., \& Akçayır, M. (2018). The flipped classroom: A review of its advantages and challenges. Computers \& Education, 126, 334-345. 
Cardona, F. \& Atarés, L. (2016). Motivación al estudio de la Fisicoquímica en el Grado de Ciencia y Tecnología de Alimentos mediante el estudio de aplicaciones. Utilización para el desarrollo y evaluación de competencias transversales. INRED 2016.

Cardona, F. \& Atarés, L. (2017). Introducción de aplicaciones de la termodinámica a la tecnología de alimentos como elemento motivador y de aprendizaje en contexto. INNODOCT 2017.

Cardona, F. \& Atarés, L. (2018). Ludificación (gamification) y exámenes on-line como elemento dinamizador y motivador del estudio. INNODOCT 2018.

Cardona, F. \& Rubio-Granero, C. (2018). Aplicaciones de la química física a la tecnología de alimentos. Motivación al estudio y desarrollo de competencias transversales. INNODOCT 2018.

Cardona, F. \& Llorens-Molina, J.A. (2019). Docencia inversa en la asignatura Fundamentos Químicos para la Ciencia y Tecnología de los Alimentos. INNODOCT 2019.

Cano-García, M.E. (2008). "La evaluación por competencias en la educación superior." en Profesorado. Revista de currículum y formación del profesorado 12, 3.

De Jong, O. (2006). "Context-based chemical education: how to improve it?" Plenary lecture presented at the 19th International Conference on Chemical Education. Seoul, Korea, 12-17 August 2006.

Durán-Aponte, E. y Durán-García, M. (2013). "Aprendizaje cooperativo en la Enseñanza de Termodinámica: Estilos de Aprendizaje y Atribuciones Causales" en Revista Estilos de Aprendizaje 11:11.

Jensen, J. L., Holt, E. A., Sowards, J. B., Ogden, T. H., \& West, R. E. (2018). Investigating strategies for pre-class content learning in a flipped classroom. Journal of Science Education and Technology, 27(6), 523-535.

Llorens-Molina, J.A. (2008). Design and Assessment of an Online Prelab Model in General Chemistry: A Case Study. Journal of the Research Center for Educational Technology, vol. 4, pp.15-31.

Llorens-Molina, J.A., Atarés, L. \& Lacuesta, R. (2014). Diseño de objetos de aprendizaje en formato polimedia para el desarrollo de actividades pre-laboratorio en química general. INRED 2014.

Llorens-Molina, J.A. (2017). La evaluación formativa: un análisis desde la perspectiva de los estudiantes. INRED 2017.

Llorens-Molina, J.A. (2017b). Diseño y aplicación de objetos de aprendizaje audiovisuales para la transición hacia la clase invertida. Jornadas Virtuales de Colaboración y Formación. Ubicuo y Social: Aprendizaje con TIC (USA-TIC) 2017.

Llorens-Molina, J.A. \& Llorens de Jaime, J.M. (2018). Screencasts in the classroom. Design and assessment. 12th Annual International Technology, Education and Development Conference (INTED) 2018.

Llorens-Molina, J.A. \& Cardona, F. (2019). Desarrollo de competencias transversales mediante la creación de screencasts por los estudiantes. INNODOCT 2019. 
Llorens-Molina, J.A. \& Cardona, F. (2019). Docencia inversa en prácticas de laboratorio. Desarrollo y autoevaluación de competencias transversales. INNODOCT 2019.

O'Flaherty, J., \& Phillips, C. (2015). The use of flipped classrooms in higher education: A scoping review. The internet and higher education, 25, 85-95.

Parker, L.L. y Loudon, G.M. (2012). Case study using online homework in undergraduate organic chemistry: results and student attitudes. Journal of Chemical Education, vol. 90, 1, p. 37-44.

Rodríguez-Sandoval, E., Vargas-Solano, E. M. \& Luna-Cortés, J. (2010). Evaluación de la estrategia de aprendizaje basado en proyectos. Educación y educadores, 13,1: 13-25.

Ryan, M. D., \& Reid, S.A. (2015). Impact of the flipped classroom on student performance and retention: A parallel controlled study in general chemistry. Journal of Chemical Education, 93(1), 13-23.

Sadler, P.M. y Good, E. (2006). The impact of self-and peer-grading on student learning. Educational assessment, vol. 11, 1, p. 1-31.

Teo, T.W., Tan, K.C.D., Yan, Y.K., Teo, Y.C., \& Yeo, L.W. (2014). How flip teaching supports undergraduate chemistry laboratory learning. Chemistry Education Research and Practice, 15(4), 550-567.

Uskoković, V. (2018). Flipping the flipped: the co-creational classroom. Research and Practice in Technology Enhanced Learning, 13:11

Verdeguer-Sancho, M., Llorens-Molina, J.A., García-Breijo, F., Roselló-Caselles, J., García-Prats, A., Rubio-Michavila, C., Vargas-Colás, M., Santamarina-Siurana M.P. (2017). Diseño de una rúbrica para la evaluación de los Trabajos Final de Grado de la Escuela Técnica Superior de Ingeniería Agronómica y del Medio Natural. INNODOCT 2017. 